\title{
El objeto de conocimiento en la investigación cualitativa: un asunto epistemológico
}

\section{The object of knowledge in qualitative research: an epistemological matter}

\section{O objeto de conhecimento na pesquisa qualitativa: um assunto epistemológico}

\author{
A. Ramírez-Elías ${ }^{a 1^{*}}$, M.I. Arbesú-García ${ }^{b_{2}}$ \\ ORCID: \\ a $0000-0002-8015-5840$ \\ b 0000-0002-4503-3143 \\ ${ }^{1}$ División de Estudios Profesionales, Escuela Nacional de Enfermería y Obstetricia, \\ Universidad Nacional Autónoma de México, Ciudad de México, México \\ ${ }^{2}$ División de Ciencias y Artes para el Diseño, Universidad Autónoma \\ Metropolitana, Unidad Xochimilco, Ciudad de México, México \\ Recibido: 25 septiembre 2018/Aceptado: 25 mayo 2019
}

\section{RESUMEN}

Introducción: Los procesos reflexivos e interrogativos necesarios para desarrollar preguntas de investigación cualitativas efectivas, pueden dar forma y dirección a un estudio, sin embargo; a menudo se subestiman. Las buenas preguntas de investigación no necesariamente producen una buena investigación, pero las preguntas mal concebidas o elaboradas probablemente crearán problemas que afectarán todas las etapas posteriores de un estudio. Objetivo: Este artículo aborda el desarrollo de preguntas de investigación y cómo los procesos de generación y refinación de preguntas son fundamentales para la conformación de un estudio cualitativo. 
Desarrollo: Generar preguntas de investigación relevantes y adecuadas requiere que el investigador preste atención no solo a las preguntas en sí mismas, sino a sus conexiones con todos los componentes del diseño; es decir, los objetivos a los que pueden responder las preguntas; las implicaciones para las preguntas relacionadas con su marco conceptual; los métodos que podría usar para responder las preguntas y las amenazas de validez que deberá abordar. Al igual que con los otros componentes del diseño, reflexionar sobre estos temas es una herramienta extremadamente útil para desarrollar sus preguntas.

Conclusiones: En los estudios cualitativos, el proceso continuo de preguntas es una parte integral en la comprensión de las vidas y perspectivas de otros. Es importante enfatizar que este tipo de investigación requiere que el investigador asuma una postura epistemológica, seleccione un método y también elija ciertas técnicas que le permitan recopilar información en el trabajo de campo.

Palabras clave: Investigación cualitativa; pregunta de investigación; diseño de investigación; México.

\section{ABSTRACT}

Introduction: Although frequently underestimated, reflexive and interrogative processes needed to develop the research questions in qualitative research, can give shape and direction to a study. Good research questions do not necessarily produce a good research study, but badly conceived or elaborated research questions can create problems which will further have impacts on all stages of the study.

Objective: This study addresses the development of research questions and how their generation and refinement processes are fundamental in the conformation of a qualitative study.

Development: Generating relevant and adequate research questions requires the researchers to give attention to the questions and their relations to all the components of the design; in other words; to the objectives, the conceptual framework, the methods used, the possible implications involved, the validity, etcetera. Reflecting on these issues is a useful tool while developing research questions.

Conclusions: In qualitative studies, the ongoing process of questioning is integral to the comprehension of the lives and perspectives of others. So, it is important to emphasize that qualitative research requires the researchers to adopt an epistemological posture by selecting methods and procedures which can support their field work information gathering. Keywords: Qualitative research; research question; research design; Mexico.

\section{RESUMO}

Introdução: Os processos reflexivos e interrogativos necessários para desenvolver perguntas de pesquisa qualitativas efetivas podem dar forma e direção a um estudo, porém frequentemente são subestimados. As boas perguntas de pesquisa não necessariamente produzem uma boa pesquisa, mas as perguntas mal concebidas ou elaboradas provavelmente criarão problemas que afetarão todas as etapas posteriores de um estudo.

Objetivo: Este artigo aborda o desenvolvimento de perguntas de pesquisa e como os processos de produção e refinamento de preguntas são fundamentais para a conformação de um estudo qualitativo.

Desenvolvimento: Gerar perguntas de pesquisa relevantes e adequadas requer que o pesquisador preste atenção não só às preguntas em si mesmas, senão a suas conexões com todos 
os componentes do desenho: quer dizer, os objetivos aos que podem responder as preguntas; as implicações para as preguntas relacionadas com seu marco conceptual; os métodos que poderia usar para responder as perguntas e as ameaças de validade que deverá abordar. Ao igual que com os outros componentes do desenho, refletir sobre estes temas é uma ferramenta extremadamente útil para desenvolver suas perguntas.

Conclusões: Nos estudos qualitativos, o processo contínuo de perguntas é uma parte integral na compreensão das vidas e perspectivas de outros. É importante enfatizar que este tipo de pesquisa, requer que o pesquisador assuma uma postura epistemológica, selecione um método e também eleja certas técnicas que lhe permitam recopilar informação no trabalho de campo.

Palavras chave: Pesquisa qualitativa; pergunta de pesquisa; desenho de pesquisa; México.

\section{INTRODUCCIÓN \\ La importancia de las preguntas de investigación}

El planteamiento inicial de un problema de investigación, desde cualquier paradigma, es de suma importancia para determinar el tema de interés y el resto de la toma de decisiones que se adoptarán en el proceso que implica una investigación. En el caso de los estudios cualitativos, la pregunta de investigación condensa aspectos teóricos, metodológicos y empíricos; pero sobre todo constituye el eje transversal del proceso de indagación ${ }^{1,2}$.

Las preguntas de investigación se relacionan con diversos aspectos en el diseño, entre otros: con el nivel de precisión que requiere lo que se pretende conocer, con la finalidad del estudio y con el nivel teórico que se busca profundizar. Dichas preguntas, es decir lo que específicamente se desea comprender, son el núcleo del diseño de investigación, ya que son el único componente que se conecta directamente con todos los demás componentes del diseño. Más que cualquier otro aspecto del diseño, las preguntas de investigación influirán y deberán responder a todas las demás partes del estudio (figura 1)3.

Por otra parte, en la investigación cualitativa las preguntas buscan recopilar información que conduce a una mayor comprensión de un fenómeno4. La investigación cualitativa es una actividad científica que da cuenta de los significados de las acciones realizadas por el otro; constituye un campo de investigación que entrecruza disciplinas, áreas y objetos de estudio5. Además, este tipo de investigación se fundamenta en un enfoque o paradigma interpretativo y naturalista del mundo, lo que significa que los investigadores cualitativos no solo estudian los fenómenos y los sujetos en sus escenarios naturales, sino que tratan de comprender, conocer e interpretar lo que se observa a partir de los significados que los otros les dan a las acciones que realizan 5 . El investigador cualitativo tiene como finalidad lograr una interpretación o descripción densa de un objeto de conocimiento6.

Este paradigma también se ocupa de aprehender aquello que los actores consideran valioso o también lo que estos consideran poco importante. Los autores representantes del enfoque interpretativo no están de acuerdo con el principio del método universal que defiende el positivismo y su capacidad de predicción vía tendencias o leyes, en donde la realidad se asume de manera general7 ${ }^{7}$ A diferencia de lo anterior, a los métodos cualitativos les interesa conocer creencias, representaciones y motivos que orientan a las personas para efectuar ciertas acciones y no otras.

Muchos investigadores buscan automáticamente metodologías cuantitativas para medir el objeto de conocimiento en cuestión, cuando quizás un enfoque cualitativo sería más apropiado para ampliar y comprender en profundidad dicho objeto. De igual manera, el investigador debe ser 


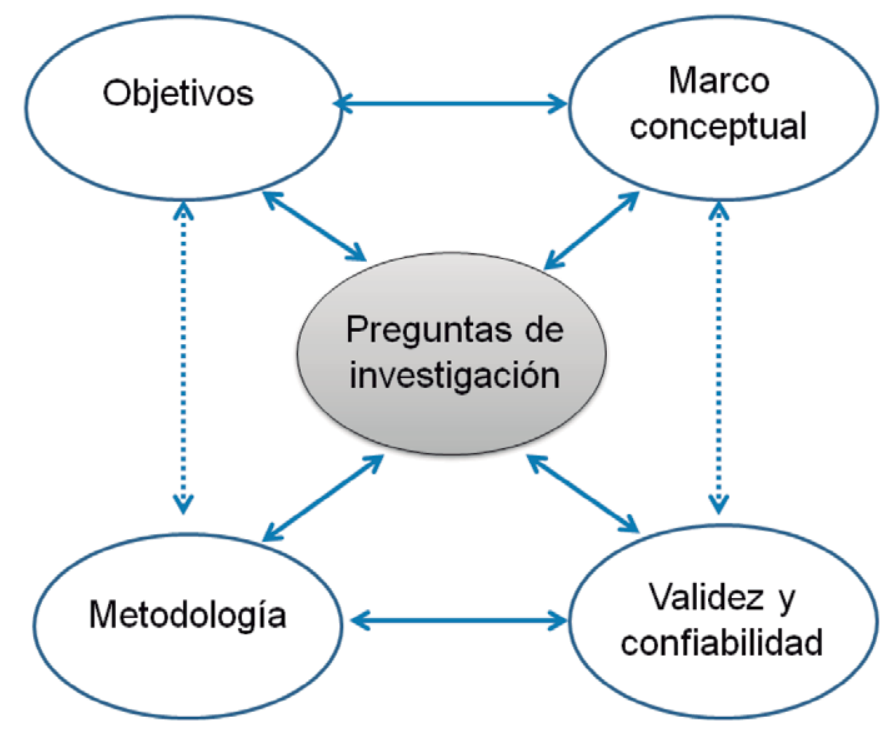

Figura 1. Relaciones e interacción de las preguntas de investigación.

cuidadoso al plantear preguntas que se puedan explorar con metodologías cualitativas, por ejemplo, estudiar en profundidad cómo da clases un profesor o qué estrategias pedagógicas utiliza para que sus alumnos aprendan; pues estas no buscan conocer de una forma general algún fenómeno o sujeto(a), más bien pretenden comprender ciertas particularidades o del por qué actúan de tal o cual forma los sujetos que forman parte del estudio, es decir cuáles son los motivos que los llevan a realizar dichas acciones.

Por ello enfatizamos que se necesitan métodos cualitativos cuando las preguntas formuladas se enfocan en indagar el por qué y el para qué de las acciones de los otros. De la misma forma que el mundo de la célula cedió a los métodos basados en la introducción del microscopio, ahora el mundo de las experiencias y los significados de los pacientes y los alumnos se está volviendo accesible a través de métodos etnográficos o fenomenológicos. El uso apropiado de la investigación cualitativa brinda nuevos conocimientos sobre la condición humana, la salud y la educación.

La investigación cualitativa es particularmente apropiada cuando se desea comprender u obtener una perspectiva personal de un evento o experiencia humana. Esta implica el uso y la recolección con una variedad de técnicas de recopilación de información como: entrevistas en profundidad, observación participante, diarios de campo, revisión de fotografías, de bitácoras, y demás técnicas que proporcionan al investigador diversos materiales empíricos que dan cuenta del por qué y para qué las personas llevan a cabo determinados actos. Cada una de estas técnicas describe minuciosamente la vida cotidiana de las personas a quienes se estudia.

Ahora bien, con el fin de poder detallar el por qué y el para qué de las acciones de los otros, existen varios métodos cualitativos o enfoques metodológicos que se pueden adoptar en una investigación de este tipo, entre otros: investigación acción, etnografía, historia de vida, estudio de caso, textos observacionales, históricos, interactivos y visuales ${ }^{5,9}$. Dichos enfoques asumen que las cosas materiales y las prácticas humanas tienen un significado, por ello, estos indagan con suma atención en los espacios donde interactúan dos o más sujetos con el propósito de describir e interpretar las experiencias de las personas, sus perspectivas e historias, o sea, estudian su cultura. 
Los investigadores cualitativos pueden identificar poblaciones para ser estudiadas y mostrar los efectos inmediatos de ciertos programas en dichas poblaciones. También crean o propician espacios en donde los sujetos estudiados (el otro), pueden expresarse; es por ello que el investigador suele ser un conducto para que las voces de los otros sean escuchadas ${ }^{5}$.

La metodología cualitativa permite a los investigadores formular diferentes preguntas de investigación, explorar y comprender fenómenos desde una perspectiva contrastante. Se abordan las preguntas qué, por qué y cómo sobre el fenómeno, en lugar de preguntas como, ¿cuál es la prevalencia de?, ¿hay una diferencia significativa entre? o ¿cuáles son los predictores más fuertes? ${ }^{9,10}$. El investigador cualitativo al plantearse las preguntas de investigación deberá cuestionarse si desea obtener una explicación detallada de cómo las personas entienden un fenómeno, en lugar de cuantificar los participantes en él.

Algunas de las preguntas de investigación a las que se ha aplicado el enfoque cualitativo incluyen las siguientes: ¿cómo enfrentan las personas la enfermedad (aguda y crónica), la adicción, la pérdida, la discapacidad, el dolor crónico, la fatiga, los tratamientos o el final de la vida?, ¿los hombres y las mujeres viven las afecciones de forma diferente?, ¿tienen diferentes percepciones de su enfermedad?, ¿qué piensan realmente acerca de los servicios de atención médica? También se han formulado preguntas para explorar el ámbito educativo tales como: ¿cuáles son las actividades de aprendizaje qué se implementan en el aula?, ¿cómo perciben los alumnos la actividad docente? Este conjunto de interrogantes centra su actividad en la ubicación de los datos más significativos que servirán después para la interpretación adecuada de los hechos o acontecimientos ${ }^{10}$.

Las historias personales pueden revelar temores y preocupaciones desconocidas sobre la práctica de la asistencia médica o el acto educativo y los eventos psicosociales relacionados. Sin embargo; como investigadores, se deberá decidir sobre el tipo de datos cualitativos que se desea reunir y cómo van a utilizarse en su posterior análisis. El investigador tendrá que preguntarse: ¿estoy particularmente interesado en el lenguaje que usan las personas para expresar sus experiencias? Si es así, es posible que desee considerar el método análisis del discurso; ¿y si simplemente desea obtener una buena descripción del rango de experiencias de un evento?; en este caso, podría considerar un análisis fenomenológico que literalmente significa describir. En su defecto, si este lo que busca es generar una descripción y teoría de los datos, es posible que el investigador desee adoptar uno de los métodos establecidos para llevar a cabo la teoría fundamentada. En todo caso, su decisión en este momento debe estar influenciada tanto por lo que quiere lograr, como por su comprensión de los antecedentes filosóficos detrás del enfoque que desea adoptar ${ }^{11}$.

Las preguntas que formulan los investigadores están influidas, explícita o implícitamente por sus experiencias personales y orientaciones filosóficas que modelan sus intereses y forma de pensar. Los investigadores pueden sentirse atraídos por una teoría concreta, dado que esta es compatible con sus marcos y preferencias conceptuales. Así, las cuestiones de una investigación surgen de distintas fuentes que coinciden parcialmente entre sí.

En las temáticas de las investigaciones, sus finalidades y orientaciones teóricas están influidas por varios factores que forman parte del propio investigador, entre los que encontramos no solo sus experiencias vitales, ideológicas, culturales y compromisos filosóficos, así como éticos; sino que también influyen las cuestiones y problemas señalados por otros miembros destacados de las comunidades científicas y de la sociedad en general. De igual manera, la reflexión sobre la propia actividad, las reacciones de los colegas y un deliberado cuestionamiento (en los ámbitos tanto empírico 
como abstracto) de ciertas premisas y supuestos implícitos, hacen posible que el investigador se haga consciente de dichas influencias y las considere de forma explícita ${ }^{1,12}$.

Por otro lado, hay factores que determinan la clarificación del propósito de la investigación, como el conocimiento de fondo existente de los investigadores (dimensión epistémica) del fenómeno particular y los intereses, motivos y preferencias del investigador (la dimensión sociológica) ${ }^{13}$.

Los investigadores cualitativos formulan preguntas y construyen respuestas que permiten destacar el modo en que la experiencia social es creada y dotada de sentido. La investigación cualitativa como proceso implica actividades con orientación genérica e interconectadas entre sí, y cubren un amplio espectro de elementos como la teoría, análisis, ontología, epistemología y metodología, entre otras. Detrás de estos términos se encuentra la biografía personal del investigador, quien habla desde una perspectiva particular de condición social, género, cultura y etnia. Desde este posicionamiento multicultural y de género, el investigador vuelve su mirada sobre el mundo con un conjunto de ideas, un cierto marco (la teoría, la ontología) que especifica una serie de interrogantes (la epistemología), que examina de un modo determinado (la metodología, el análisis). Es decir, cada investigador habla desde una comunidad interpretativa peculiar, que le es propia y que configura, a su manera, los componentes culturales y genéricos del acto de investigación. No existen, por lo tanto, observaciones objetivas, sino sólo observaciones situadas socialmente en (y entre) los mundos del sujeto observador y el observado $0^{6,10,14}$.

Estas creencias dan forma al modo en que el investigador cualitativo ve el mundo y actúa en él. El investigador se encuentra siempre aferrado a una red de premisas epistemológicas y ontológicas que, más allá de su verdad o falsedad en términos absolutos, tienen parcialmente carácter autovalidatorio. La red que contiene estas premisas epistemológicas, ontológicas y metodológicas puede recibir el nombre de paradigma o marco interpretativo, un conjunto básico de creencias que guían la acción. Cada paradigma interpretativo le manifiesta demandas específicas al investigador, e incluye las preguntas que se formula y las interpretaciones que da de ellas ${ }^{1,11}$.

Elaborar preguntas de investigación significativas es una habilidad que va más allá de la mecánica de la construcción de preguntas, para inclinarse hacia una comprensión más holística de los problemas de investigación cualitativa. Por lo tanto, en lugar de ver el proceso de creación y construcción de preguntas de investigación como una tarea mecánica, sostenemos que es un oficio que debe aprenderse en contextos específicos; y requiere un estudio y reflexión continúa. Además, se basa en la comprensión de los investigadores de las historias y las tradiciones de la investigación cualitativa, así como su conocimiento sobre los enfoques existentes ${ }^{15,16}$.

\section{DESARROLLO}

\section{Interacciones entre las preguntas de investigación y la teoria}

Incluso sin una gran conciencia teórica, las teorías subyacentes siempre estarán presentes pues guían la mirada del investigador. Los científicos hacen su investigación en un contexto de teoría, basado en paquetes unitarios de creencias, denominados paradigmas. La teoría está inextricablemente ligada a las preguntas de investigación, ya sea que la teoría las forme inicialmente o sugiera nuevas preguntas a medida que se desarrolla el estudio. Una pregunta general para muchos estudios cualitativos, apuntará hacia uno o más de los constructos teóricos que enmarcan el estudio.

El concepto de paradigma es más general que una teoría. Se refiere a las principales suposiciones sobre la visión del mundo y el conocimiento, no sólo indica una actitud comúnmente aplicada o un área temática, sino que los paradigmas abarcan tanto la ontología como la epistemología. La 
ontología se refiere a la naturaleza del ser o cómo son básicamente las cosas, mientras que la epistemología trata de la naturaleza del conocimiento o el estudio de ciertos aspectos del ser. Estos dos dominios están lógicamente entrelazados en cómo entendemos que el mundo determina la adecuación del enfoque de estudio. La consistencia entre la ontología y la epistemología es esencial en la investigación ${ }^{17}$.

Las preguntas de investigación conforman el inicio del proceso, por ende, son un componente importante a partir del cual se genera la producción de conocimiento ${ }^{12}$. El principio que las fundamenta es la necesidad de producir conocimientos a través del contacto directo con los sujetos investigados, en escenarios en los cuales tiene lugar la confección de significados sociales, culturales y personales para poder descubrir o reconocer: los conflictos y fracturas; las divergencias y consensos; las regularidades e irregularidades; las diferencias y homogeneidades que caracterizan la dinámica subyacente al objeto de conocimiento ${ }^{15,16}$.

La pregunta de investigación se enmarca en una teoría, en esta estrecha simbiosis ocurre la selección de fenómenos relevantes, la interpretación de los testimonios y posibles explicaciones de las causas o influencias subyacentes de los referentes empíricos observados. Para la investigación cualitativa, las teorías son especialmente importantes como herramientas para comprender, interpretar y elaborar observaciones empíricas más allá de la descripción ${ }^{17}$.

Escribir las preguntas de un estudio cualitativo es desafiante, se relaciona con la congruencia metodológica y la inexorable interconexión entre preguntas de investigación, propósitos y métodos. Los diseños de investigación deberán ser metodológicamente congruentes con preguntas cuidadosamente desarrolladas; tal congruencia también podría guiar la elección de métodos y técnicas particulares, así como orientar para la implementación del diseño de investigación ${ }^{16}$. Los métodos son el componente más flexible, pragmático e intrínsecamente teórico del proceso de investigación, fuertemente influenciado por la metodología (a través de los objetivos, preguntas de investigación y diseño de estudio) y la epistemología. También son el camino hacia el producto final de la investigación ${ }^{13,18}$.

Los problemas y las preguntas de investigación son influenciados por la teoría, la cual actúa en el proceso de investigación como esquema organizador ${ }^{17}$. En dicho proceso se identifican tres componentes: a) Preconcepción, b) Comprensión actual, c) Interpretación; elementos que configuran una estructura dialéctica de conocimientos y constituyen la base de los procesos de investigación. La interpretación parte de una preconcepción de lo que se pretende interpretar ${ }^{19,20}$.

La teoría en algunos casos determina la formulación de las preguntas; puede suceder que en estas se planteen ciertas cuestiones con el fin de ampliar, perfeccionar o verificar teorías establecidas. A veces se trata de la comprobación rigurosa de una teoría existente, y otras más, de la indagación exploratoria mediante la que se pretende averiguar si un fenómeno concreto logra explicarse a través de una nueva teoría1. Muchos investigadores cualitativos deciden replantear la indagación en ciertas áreas porque el marco teórico existente no captura adecuadamente ni refleja los datos empíricos; por consiguiente, el diseñó cualitativo se enfoca a subsanar esta brecha cuando es poca la literatura que describe las diferencias y por qué podría ocurrir un fenómeno determinado8.

Las preguntas de investigación cuidadosamente formuladas, permiten que los investigadores cualitativos cuenten con elementos sólidos que les permitan elegir el diseño de investigación, ayudándolos a considerar la idoneidad de diversas posibilidades epistemológicas y metodológicas ${ }^{16}$. Cualquiera que sea el caso, el punto de partida es asumir una posición epistemológica por el investigador, ya que es fundamental y esta influirá directamente en los métodos y la metodología. Es 
probable que esta posición se encuentre determinada por la disciplina de la cual este provenga, así como de las teorías formales de conocimiento que el investigador haya leído ${ }^{9,18}$. También es probable que las respuestas a las preguntas de investigación sean consistentes con los supuestos paradigmáticos y epistemológicos del estudio. De esta manera, las preguntas de investigación apoyan a los investigadores a medida que eligen las decisiones adecuadas ${ }^{16}$.

Las decisiones sobre epistemología son importantes porque influirán en la elección de la metodología, dado que algunas epistemologías y metodologías son inconmensurables. La epistemología también limitará la práctica de la investigación (método), pues determina las relaciones entre el investigador y el participante, las medidas apropiadas de la forma en que se construye el conocimiento y la naturaleza de la información. En términos simples, la metodología justifica el método, que produce información y posterior análisis. El conocimiento se crea a partir de los fenómenos y análisis. La epistemología modifica la metodología y justifica el conocimiento producido o,18.

Los investigadores suelen afirmar que utilizan la metodología que mejor se adapte a los objetivos del estudio y la pregunta de investigación, esta relación funciona en dos direcciones. Los objetivos, las preguntas de investigación y el diseño determinan la elección de la metodología y esta conforma los objetivos, las preguntas de investigación y el diseño ${ }^{18}$.

La contribución epistemológica en la investigación es esencialmente teórica, ya que tiene que ver con las teorías del conocimiento, esta acción es ineludible. Mientras un investigador reflexivo adopta activamente una teoría del conocimiento, uno menos reflexivo adopta implícitamente una teoría del conocimiento, puesto que es imposible participar en la creación de conocimiento sin al menos suposiciones tácitas sobre qué es el conocimiento y cómo se construye ${ }^{16,18}$.

\section{La problematización del tema}

El primer paso en una propuesta de investigación es seleccionar un tema que requiera ser investigado, este puede provenir de una lista de temas que son nuevos, de problemas encontrados en la práctica diaria o de áreas de impacto emergentes. En esta etapa incipiente del proceso de investigación tenemos un tema, hemos identificado un objeto de estudio, ¿pero tenemos un problema de investigación?

En relación con este último, se entiende por problematizar la acción orientada a reelaborar el objeto de conocimiento de manera que sea posible captar bajo determinadas coordenadas espaciotemporales la complejidad que se condensa en el problema, aquella que emerge del análisis concreto de un sector de la realidad social o cultural. Es decir, reelaborar prácticas, discursos y pensamientos en torno al sujeto-objeto, no conocidos de antemano (por ende, no abarcados en el planteamiento inicial), que se constituyen así en problemas para la disciplina que los plantea ${ }^{12,15}$.

Un diseño de investigación apropiado ofrece un modo coherente de abordar el asunto o el problema, esto explica por qué resulta importante conocer a fondo el tipo de tema a tratar. Distintos tipos de cuestiones o problemas necesitarán diferentes tipos de diseños de investigación. En una investigación cualitativa, la selección de los tópicos de investigación y la conceptualización de los mismos, se hace a través del contacto directo con una manifestación concreta de una realidad humana, social o cultural ${ }^{15,21}$. Este proceso compromete por lo menos tres aspectos decisivos en la investigación:

a. Al investigador: en la problematización, éste se cuestiona a sí mismo y a su capacidad de interrogar un conjunto de fenómenos y sus relaciones.

b. Al sujeto-objeto de estudio: en la problematización el investigador avanza en la clarificación del sujeto-objeto de estudio; busca la trama problemática en que el objeto aparece 
objetivado de múltiples formas aparentemente desarticuladas, descontextualizadas, aisladas y en cuya operación de articulación encontrará que por debajo de los problemas, invisibilizado, está el campo problemático que tiene que revelar y en cuyo espacio el problema adquiere sentido para la disciplina.

c. Al problema de investigación que se convierte en proceso de problematización: abarca todo el desarrollo de la investigación, el que implica un proceso de construcción gradual del problema, de inserción del problema en el campo problemático que oficia de contexto ${ }^{12,20}$.

\section{Características de las preguntas de investigación}

Existe un acuerdo tácito en que los buenos estudios cualitativos responden preguntas de investigación importantes y claramente formuladas. Una adecuada pregunta de investigación hace la diferencia en la profundidad de los hallazgos y las aportaciones al campo de estudio. En algunos casos, formular una pregunta apropiada al comienzo de un estudio puede ser relativamente sencillo. Esto ocurre cuando existen marcos teóricos y conceptuales bien desarrollados, y por lo tanto se ha estudiado ampliamente el tema. La literatura de investigación existente puede señalar áreas donde se necesita más investigación ${ }^{2,8}$.

Cuanto menor sea la claridad con la que se formulen las preguntas de investigación, mayor es el peligro de que los investigadores se encuentren al final ante montañas de datos intentando en vano interpretarlos. Es importante que el investigador desarrolle ideas claras de las preguntas que busca responder, para comprobar la conveniencia de las decisiones metodológicas. La formulación precisa de la pregunta de investigación es un paso central al conceptualizar el diseño de investigación. Estas preguntas constituyen un punto de referencia para comprobar la solidez del diseño y la conveniencia de los métodos realizados para recoger e interpretar los datos 3.

Podríamos afirmar que las preguntas de investigación son como una puerta al campo de investigación que esté en estudio. Que las actividades empíricas investigadas produzcan respuestas o no, depende de la formulación de dichas preguntas; también depende de esto la decisión sobre qué métodos son apropiados y quiénes (es decir, qué personas, grupos o instituciones) o qué (es decir, qué procesos, actividades, estilos de vida) se deberían de incluir en el estudio.

Las preguntas de investigación se pueden clasificar al tener en cuenta hasta qué punto son idóneas para confirmar o refutar supuestos existentes o hasta qué punto pretenden descubrir supuestos nuevos, o al menos permitir esto ${ }^{22}$. Una investigación correctamente formulada suele aclarar los propósitos y la pregunta de investigación, lo que llevará a seleccionar los métodos apropiados. En general, esto es más sólido cuando el investigador puede demostrar que ha establecido métodos mediante referencia a un marco teórico ${ }^{9,23}$.

Existe un peligro real para el estudio si no se formulan cuidadosamente las preguntas de investigación en relación con los otros componentes del diseño. Las preguntas de investigación deben contener lo que se desea lograr al realizar el estudio (los objetivos), y lo que ya se conoce sobre las cosas que se estudiará y sus teorías tentativas sobre estos fenómenos (el marco conceptual). No hay ninguna razón para plantear preguntas de investigación para las cuales las respuestas ya están disponibles, que no se conectan de manera clara con lo que cree que está sucediendo realmente, o que no tienen relevancia directa para sus objetivos al realizar la investigación³.

Los modelos de diseño que colocan la formulación de preguntas de investigación al comienzo del proceso, y que ven estas preguntas como determinantes de otros aspectos del diseño, no hacen justicia al carácter interactivo e inductivo de la investigación cualitativa. Las preguntas de investigación 
en un estudio cualitativo no deben formularse en detalle hasta que se aclaren los objetivos y el marco conceptual (y, a veces, los aspectos generales del muestreo y la recopilación de datos) del diseño, deben permanecer sensibles y adaptables a las implicaciones de otras partes de este. A menudo, se tendrá que hacer una parte significativa de la investigación antes de que quede claro qué preguntas específicas de investigación tiene sentido tratar de responder. Es decir, pensar en un plan flexible o emergente, que orientará tanto el contacto con la realidad humana objeto de estudio, como la manera en que se construirá el conocimiento acerca de ella3,24,25.

Algunas de las consideraciones que se debe tomaren cuenta cuando se formula(n) pregunta(s) de investigación de tipo cualitativa son:

- Considerar que al inicio de la pregunta se incluyan las palabras qué, cómo, por qué. Aunque parezca simple, cada palabra hace la diferencia al dar forma a lo que se está preguntando exactamente.

- Evitar realizar suposiciones. Las palabras mismas están cargadas de suposiciones ya hechas. La investigación cualitativa no busca necesariamente definir términos como en un estudio cuantitativo (definiciones operacionales), sino que contiene palabras abiertas para que el significado no esté dado por sentado, sino que pueda ser cuestionado y desarrollado.

- Realizar preguntas breves y emplear hasta donde sea posible, conceptos descriptivos. Las preguntas tienen que estar redactadas de una manera clara y directa, pero al mismo tiempo, necesitan precisar qué es lo que se está tratando de investigar. Recurrir a preguntas de investigación muy simples: como ¿cuál es el significado de tal cosa?

- Reconocer que las preguntas de investigación son la parte más importante de la investigación es la luz que guía todo el estudio y cada paso en el proyecto. Por lo tanto, considerar que debe haber congruencia entre las preguntas de investigación y la metodología específica. Es realmente fácil distraerse, seguir otras rutas interesantes, pero uno siempre tiene que ser fiel a la(s) pregunta(s).

- La conclusión de un estudio de investigación cualitativo responde a la(s) pregunta(s) planteada(s), es decir, al vacío de conocimiento que el desarrollo de la investigación pretende llenar.

- Identificar claramente el fenómeno de interés. Determinar a qué o quién se dirige la(s) pregunta(s). Estas adquieren una estructura o forma diferente y dependen de qué posición adopte el investigador en relación con el tema o problema. Entonces, una pregunta de investigación conlleva una gran cantidad de información; pues se trata de una pregunta que capta cómo, quién y qué quieres explorar ${ }^{23,24 .}$

\section{CONCLUSIONES}

Como se ha señalado en este documento, la investigación cualitativa se fundamenta en un enfoque o paradigma interpretativo y naturalista del mundo. En este sentido, los investigadores cualitativos estudian ciertos fenómenos o sujetos en sus escenarios naturales. El interés se focaliza en indagar, comprender e interpretar lo que se observa a partir de los significados que los otros les dan a las acciones que realizan. El investigador cualitativo tiene como finalidad lograr una interpretación o descripción densa de un objeto de conocimiento. El paradigma cualitativo asume que las cosas materiales y las prácticas humanas tienen un significado, por ello, indaga con suma atención en los espacios 
donde interactúan dos o más sujetos, con el propósito de describir e interpretar las experiencias de las personas, sus perspectivas e historias, en otras palabras, estudia su cultura.

Así mismo en este artículo, desde el enfoque cualitativo se aborda el planteamiento de las preguntas de investigación y cómo los procesos de generación y refinación de estas preguntas son fundamentales para la conformación de un estudio cualitativo. Dichas preguntas representan un vacío de conocimiento que el investigador tiene que llenar conforme se va desarrollando el proceso de la investigación; estas conforman el inicio del proceso y, por ende, son un componente importante a partir del cual se genera la producción de conocimiento a través del contacto directo con los sujetos investigados, en los escenarios en los cuales tiene lugar la confección de significados sociales, culturales y personales. Por consiguiente, una adecuada pregunta de investigación hace la diferencia en la profundidad de los hallazgos y las aportaciones al campo de estudio.

Los investigadores cualitativos formulan las preguntas y construyen respuestas a partir de las cuales, dan cuenta de una experiencia social dotada de sentido. En los estudios de tipo cualitativo, con frecuencia se cuestionan el por qué y el para qué los sujetos o los fenómenos estudiados realizan determinadas acciones. Más aún, las preguntas tienen relación con todas las demás partes constitutivas del diseño de la investigación. Por ello, generar preguntas de investigación relevantes y correctamente formuladas requiere que el investigador preste atención no sólo a estas en sí mismas, sino también a todos los componentes del diseño, es decir, a los objetivos que pueden responder las preguntas; a las implicaciones que tienen éstas en relación con el marco conceptual; a los métodos que se podrían usar para responder dichas preguntas; $\mathrm{y}$ a las cuestiones relacionadas con la credibilidad del estudio.

Por último, deseamos que las personas que lean este artículo cuenten con un documento sólidamente sustentado, el cual les permita entender la importancia que tiene en los estudios de tipo cualitativo, plantear con suma precisión las preguntas de investigación, a partir de las cuales se inicia el diseño de una investigación.

\section{RESPONSABILIDADES ÉTICAS}

Protección de personas y animales. Las autoras declaran no haber realizado experimentos en seres humanos ni en animales para el desarrollo de esta investigación, ya que se trata de una de tipo documental.

Financiamiento. Ninguno.

Conflicto de interés. Las autoras declaran no tener conflicto de interés.

\section{REFERENCIAS}

1. Goetz JP, LeCompte MD. Etnografía y diseño cualitativo en investigación educativa. Madrid: Morata; 1988.

2. Hamui-Sutton A. La pregunta de investigación en los estudios cualitativos. Investigación educ. médica. 2016; 5(17): 49-54. http://dx.doi.org/10.1016/j.riem.2015.08.008

3. Maxwell JA. Designing a Qualitative Study. En: Hedrick TE, Bickman L, Rog DJ. Applied research designs. A practical guide. London: SAGE; 2008. http://dx.doi.org/10.4135/9781412983457

4. Macfarlane MD, Kisely S, Loi S, Looi JC, Merry S, Parker S, et al. Getting started in research: research questions, supervisors and literature reviews. Australas Psychiatry. 2015; 23(1): 8-11.

https://doi.org/10.1177/1039856214553315 
5. Denzin NK, Lincoln YS. Manual de investigación cualitativa. Volumen 1. El campo de la investigación cualitativa. Barcelona: Gedisa; 2012.

6. Geertz C. El antropólogo como autor. Barcelona: Paidós; 1989.

7. Hammersley M, Atkinson P. Etnografía. Métodos de investigación. $2^{a}$ ed. Barcelona: PAIDOS; 1994.

8. Frankel RM, Devers KJ. Study design in qualitative research—1: Developing questions and assessing resource needs. Educ Health. 2000; 13(2): 251-261. https://doi.org/10.1080/13576280050074534

9. Kemparaj U, Chavan S. Qualitative research: a brief description. Indian J Med Sci. 2013; 67(3-4): 8998. https://doi.org/10.4103/0019-5359.121127

10. Martínez-Miguélez M. Ciencia y arte en la metodología cualitativa. 2da. Edición, México; Trillas. 2015.

11. Hale ED, Treharne GJ, Kitas GD. Qualitative methodologies I: asking research questions with reflexive insight. Musculoskeletal Care. 2007; 5(3): 139-47. https://doi.org/10.1002/msc.109

12. Quiñones $M$, Supervielle $M$, Acosta $M J$. Introducción a la sociología cualitativa: fundamentos epistemológicos y elementos de diseño y análisis. $2^{\text {a }}$ ed. Uruguay: Unidad de Comunicación de la Universidad de la República-UCUR; 2017.

13. Klopper H. The qualitative research proposal. Curationis. 2008; 31(4): 62-72. https://bit.ly/3oCgiUo

14. Martínez C, Otálvaro JC, Jáider C. La elección de la postura epistemológica del investigador y sus consecuencias metodológicas, éticas y prácticas. Revista Facultad Nacional de Salud Pública. 2015; 33(supl 1): S81-3. https://doi.org/10.17533/udea.rfnsp.v33s1a13

15. Quintana A, Montgomery W. Psicología: tópicos de actualidad. Lima: UNMSM; 2006.

16. Koro-Ljungberg M, Hayes S. Proposing an argument for research questions that could create permeable boundaries within qualitative research. J Ethnogr Qual Res. 2010; 4(3): 114-24. https://bit.ly/2ZuWgxi

17. Malterud K. Theory and interpretation in qualitative studies from general practice: Why and how? Scand J Public Health. 2016; 44(2): 120-9. https://doi.org/10.1177/1403494815621181

18. Carter SM, Little M. Justifying knowledge, justifying method, taking action: epistemologies, methodologies, and methods in qualitative research. Qual Health Res. 2007; 17(10): 1316-28. https://doi.org/10.1177/1049732307306927

19. Quecedo R, Castaño C. Introducción a la metodología de investigación cualitativa. Rev psicodidáctica. 2002; (14): 5-39. https://bit.ly/2d51Zh3

20. Agee J. Developing qualitative research questions: a reflective process. Int J Qual Stud Educ. 2009; 22(4): 431-47. https://doi.org/10.1080/09518390902736512

21. Lankshear C, Knobel M. Problemas asociados con la metodología de la investigación cualitativa. Perf. educ. 2000; 22(87): 6-27. https://bit.ly/2LhrbUy

22. Flick U. Introducción a la investigación cualitativa. Madrid: Morata; 2004.

23. Meyrick J. What is good qualitative research? A first step towards a comprehensive approach to judging rigour/quality.J Health Psychol. 2006;11(5): 799-808. https://doi.org/10.1177/1359105306066643

24. Smythe L, Giddings LS. From experience to definition: addressing the question 'what is qualitative research?' Nurs Prax N Z. 2007; 23(1): 37-57. https://bit.ly/30DFyJj

25. Sandoval-Casilimas CA. Investigación cualitativa. Bogotá: Instituto Colombiano para el Fomento de la Educación Superior; 1996. https://bit.ly/2ip3fkv 